\title{
Ghost Cohomologies and Hidden Space-Time Symmetries
}

\author{
Dimitri Polyakov ${ }^{\dagger}$ \\ Center for Advanced Mathematical Studies \\ and Department of Physics \\ American University of Beirut \\ Beirut, Lebanon
}

\begin{abstract}
We observe and study new non-linear global space-time symmetries of the full ghost+matter action of $R N S$ superstring theory. We show that these surprising new symmetries are generated by the special worldsheet currents ( physical vertex operators) of RNS superstring theory, violating the equivalence of superconformal ghost pictures. We review the questions of BRST invariance and nontriviality of picture-dependent vertex operators and show their relation to hidden space-time symmetries and hidden space-time dimensions. In particular, we relate the space-time transformations, induced by picture-dependent currents, to the symmetries observed in the $2 \mathrm{~T}$ physics approach.
\end{abstract} PACS:04.50. $+h ; 11.25 . M j$.

January 2007

\footnotetext{
$\dagger$ dp02@aub.edu.lb
} 


\section{Introduction}

In RNS superstring theory the physical states are described by BRST-invariant and nontrivial vertex operators, corresponding to various string excitations. Typically, these operators are defined up to transformations by the picture-changing operator $\Gamma=\left\{Q_{b r s t}, \xi\right\}$ and its inverse $\Gamma^{-1}=-4 c \partial \xi e^{-2 \phi}$ where $\xi=e^{\chi}$ and $\phi, \chi$ is the pair of the bosonized superconformal ghosts: $\beta=\partial \xi e^{-\phi}$ and $\gamma=e^{\phi-\chi}$. Acting with $\Gamma$ or $\Gamma^{-1}$ changes the ghost number of the operator by 1 unit, therefore each perturbative string excitation (such as a photon or a graviton) can be described by an infinite set of physically equivalent operators differing by their ghost numbers, or the ghost pictures. Typically, for a picture $n$ operator one has

$$
: \Gamma V^{(n)}:=V^{(n+1)}+\left\{Q_{b r s t}, \ldots\right\}
$$

and

$$
: \Gamma^{-1} V^{(n)}:=V^{(n-1)}+\left\{Q_{b r s t}, \ldots\right\}
$$

The inverse and direct picture-changing operators satisfy the OPE identity

$$
\begin{array}{r}
\Gamma(z) \Gamma^{-1}(w)=1+\left\{Q_{b r s t}, \Lambda(z, w)\right\} \\
\Lambda(z, w)=: \Gamma^{-1}(z)(\xi(w)-\xi(z)):
\end{array}
$$

The standard perturbative superstring vertices can thus be represented at any integer (positive or negative) ghost picture. Such a discrete picture changing symmetry is the consequence of the discrete automorphism symmetry in the space of the supermoduli [1], [2]. Varying the location of picture-changing operator (or, equivalently, varying the super Beltrami basis) inside correlation functions changes them by the full derivative in the supermoduli space. This ensures their picture invariance after the appropriate moduli integration, if the supermoduli space has no boundaries or global singularities. The global singularities, however, do appear in case if a correlation function contains vertex operators $V\left(z_{n}\right)$ such that the supermoduli coordinates diverge faster than $\left(z-z_{n}\right)^{-2}$ when they approach the insertion points on the worldsheet [2]. If the latter is the case, the moduli integration of the full derivative term would result in the nonzero boundary contribution and the correlation function would be picture-dependent. The first important physical example of how picture-dependent operators emerge in superstring theory is the following. 
Consider NSR superstring theory (critical or noncritical) in $d$ dimensions. Its wordlsheet action is given by

$$
S=\frac{1}{2 \pi} \int d^{2} z\left\{-\frac{1}{2} \partial X_{m} \bar{\partial} X^{m}-\frac{1}{2} \psi_{m} \bar{\partial} \psi^{m}-\frac{1}{2} \bar{\psi}_{m} \partial \bar{\psi}^{m}+b \bar{\partial} c+\bar{b} \partial \bar{c}+\beta \bar{\partial} \gamma+\bar{\beta} \partial \bar{\gamma}\right\}
$$

where, for a time being, we have skipped the Liouville part. This action is obviously invariant under two global $d$-dimensional space time symmetries - Lorenz rotations and translations. It does have, however, yet another surprising and non-trivial space-time symmetry, which, to our knowledge, has not been discussed in the literature so far. That is, one can straightforwardly check that the action (2) is invariant under the following non-linear global transformations, mixing the matter and the ghost sectors of the theory:

$$
\begin{array}{r}
\delta X^{m}=\epsilon\left\{\partial\left(e^{\phi} \psi^{m}\right)+2 e^{\phi} \partial \psi^{m}\right\} \\
\delta \psi^{m}=\epsilon\left\{-e^{\phi} \partial^{2} X^{m}-2 \partial\left(e^{\phi} \partial X^{m}\right)\right\} \\
\delta \gamma=\epsilon e^{2 \phi-\chi}\left(\psi_{m} \partial^{2} X^{m}-2 \partial \psi_{m} \partial X^{m}\right) \\
\delta \beta=\delta b=\delta c=0
\end{array}
$$

The accurate proof of this fact will be given in the section 3 of this paper. Given the transformations (3),it is not difficult to check that their generator is given by

$$
T=\int \frac{d z}{2 i \pi} e^{\phi}\left(\partial^{2} X_{m} \psi^{m}-2 \partial X_{m} \partial \psi^{m}\right)
$$

The integrand of (4) is a primary field of dimension 1, i.e. a physical generator. While it is not manifestly BRST-invariant (it doesn't commute with the supercurrent terms of $\left.Q_{b r s t}\right)$ below it will be demonstrated how its BRST invariance can be restored by adding the appropriate b-c ghost dependent terms. The peculiar property of this generator is that it is annihilated by $\Gamma^{-1}$ and has no analogues at higher pictures, such as $0,-1$ and -2 . There is, however, a picture -3 version of this generator, with the manifest BRST invariance. This version can be obtained simply by replacing $e^{\phi} \rightarrow e^{-3 \phi}$ in (4). Similarly to the picture +1 -version, the picture -3 version is annihilated by $\Gamma$, so the operator is picture-dependent. We will return to the operators of the type (4), as well as to the surprising symmetry (3), in the next sections of the paper, and for now will consider the next important example of picture-dependent vertex operators in critical RNS superstring theory,given by the five-form state

$$
V_{5}(k)=H(k) \oint \frac{d z}{2 i \pi} U_{5}(z) \equiv H_{m_{1} \ldots m_{5}}(k) \oint \frac{d z}{2 i \pi} e^{-3 \phi} \psi^{m_{1}} \ldots \psi^{m_{5}} e^{i k X}(z)
$$


with the five-form $H$ subject to the BRST non-triviality condition

$$
k_{\left[m_{6}\right.} H_{\left.m_{1} \ldots m_{5}\right]}(k) \neq 0
$$

or simply $d H \neq 0$. That is, if the condition (6) isn't satisfied, there exists an operator

$$
S_{5}=H_{m_{1} \ldots m_{5}}(k) \oint \frac{d z}{2 i \pi} \partial \xi e^{-4 \phi} \psi^{m_{1}} \ldots \psi^{m_{5}}\left(\psi^{m} \partial X_{m}\right) e^{i k X}
$$

which commutator with $Q_{b r s t}$ is $V_{5}$, i.e. the five-form (5) is trivial. Indeed, if the $H$ form is closed, the $S_{5}$ operator (7) is the primary field and its commutator with $\gamma \psi \partial X$ term of the BRST charge gives $V_{5}$. If, however, the condition (6) is satisfied, $S_{5}$ doesn't commute with the stress tensor $c T$ term of $Q_{b r s t}$ and therefore $V_{5}$ cannot be represented as a commutator of $Q$ with $S_{5}$ or any other operator, as we will show by explicit computation of its correlators. The non-triviality condition (6) has a natural physical interpretation. It simply implies that all the nonzero scattering amplitudes involving the $V_{5}$-operator (5) must be the functions of $d H$ rather than $H$ itself; accordingly, they vanish for the closed $H$-forms when the $V_{5}$-operator becomes trivial. This $V_{5}$ operator is annihilated by the picture-changing transformation and the supermoduli approaching $U(z)$ diverge as $(z-w)^{-4}$ which can be read off the OPE of $U$ with the worldsheet supercurrent:

$$
G(w) U_{5}(z) \sim(z-w)^{-4} c \xi e^{-4 \phi} \psi^{m_{1}} \ldots \psi^{m_{5}} e^{i k X}+\ldots
$$

As it will be shown below, the correlation functions involving the operators of the $V_{5}$-type are picture-dependent and in fact this is one of the direct consequences of (8). The $V_{5^{-}}$ operator of (5) is an example of the ghost-matter mixing operator as its dependence on the superconformal ghost degrees of freedom can't be removed by the picture-changing and therefore isn't just an artifact of the gauge. This operator exists at all the negative pictures below -3 , but has no version at pictures $-2,-1$ or zero. At the first glance the existence of such a physical operator seems to contradict our standard understanding of the picture-changing, implying that all the vertices must exist at all equivalent pictures. So the first suspicion is that the $V_{5}$-state is BRST-trivial, as the relation (1) seems to imply that all the BRST-invariant operators annihilated by picture-changing can be written as $\left[Q_{b r s t}, S\right]$ for some S. Indeed, if $V_{5}$ is annihilated by $\Gamma$, using (1) and the invariance of $V_{5}$, we have

$$
0 \equiv \lim _{w \rightarrow z} \Gamma^{-1}(u) \Gamma(w) V_{5}(z)=1+\lim _{w \rightarrow z}\left\{Q_{b r s t}, \Lambda(u, w) V_{5}(z)\right\}
$$


and hence $V_{5}$ is the BRST commutator:

$$
V_{5}(z)=-\lim _{w \rightarrow z}\left\{Q_{b r s t}, \Lambda(u, w) V_{5}(z)\right\}
$$

This commutator can be written as

$$
\begin{array}{r}
\lim _{w \rightarrow z}\left\{Q_{b r s t}, \Lambda(u, w) V_{5}(z)\right\}=\lim _{w \rightarrow z}\left\{Q_{b r s t}, \Gamma^{-1}(u)(\xi(w)-\xi(u)) V_{5}(z)\right\} \\
=\lim _{w \rightarrow z} \Gamma^{-1}(u)(\Gamma(w)-\Gamma(u)) V_{5}(z)
\end{array}
$$

where we used $\left\{Q_{b r s t}, \xi\right\}=\Gamma$ and $\left[Q_{b r s t}, \Gamma^{-1}\right]=0$. But since $V_{5}$ is annihilated by $\Gamma$ at coincident points,

$$
\lim _{w \rightarrow z} \Gamma^{-1}(u) \Gamma(w) V_{5}(z)=0
$$

and the commutator (10) is given by

$$
V_{5}(z)=\left\{Q_{b r s t}, \xi \Gamma^{-1}(u) V_{5}(z)\right\}
$$

Thus $V_{5}$ is indeed the BRST commutator, but with an operator outside the small Hilbert space and therefore (1) does not by itself imply its triviality. This point is actually quite a conceptual one. The very possibility of having BRST non-trivial and invariant operators annihilated by $\Gamma$ (or similarly, by $\Gamma^{-1}$ ) follows from the fact that the bi-local $\Lambda(u, w)$ operator (which, by itself, is in the small Hilbert space) vanishes at coincident points. Had this not been the case, (1) would have implied that any operator $V$, annihilated by $\Gamma$, would have been given by the commutator $V(z)=-\left\{Q_{b r s t}, \Lambda(z, z) V\right\}$, i.e. would have been BRST exact. But since $\Lambda(z, z)=0$, the OPE (1) only leads to the trivial identity of the type $V=V$, as it is clear from (13) (note that $\left\{Q_{b r s t}, \xi \Gamma^{-1}\right\}=1$ ) The picturedependent $V_{5}$ operator (5) is actually BRST non-trivial and invariant, i.e. it is a physical state. In fact, the $V_{5}$-operator is not related to any point-like perturbative string excitation but to the D-brane dynamics [3], and its non-perturbative characted is somehow encripted in its non-trivial picture dependence. While the questions of its non-triviality have been discussed previously (see, e.g. [3]), below we shall review the standard arguments leading to the standard concept of picture equivalence of physical states (which indeed is true for the usual perturbative point-like string excitations like a graviton or a photon) and show where precisely these arguments fail for the case of $V_{5}$. So let us further analyze the question of the nontriviality of picture-dependent operators. The next standard argument for the picture-equivalence stems from the fact that one is able to freely move the picture-changing 
operators inside the correlators. Indeed, since the derivatives of $\Gamma$ are all BRST-trivial: $\partial^{n} \Gamma=\left\{Q_{b r s t}, \partial^{n} \xi\right\} ; n=1,2, \ldots$ one can write

$$
\Gamma(w)=\Gamma(z)+\left\{Q_{b r s t}, \sum_{n} \frac{(w-z)^{n}}{n !} \partial^{n} \xi\right\} .
$$

Next, using the identity $1=: \Gamma \Gamma^{-1}$ :, following directly from (1), one can write for any correlator including $V_{5}$ and some other physical operators $U_{i}\left(z_{i}\right), i=1, \ldots n$

$$
<V_{5}(z) U_{1}\left(z_{1}\right) \ldots U_{n}\left(z_{n}\right)>=<\Gamma^{-1} \Gamma(w) V_{5}(z) U_{1} \ldots U_{n}>
$$

for any poinnt $w$. Using (14) one can move $\Gamma$ from $w$ to $z$ but, since $: \Gamma U_{5}:(z)=0$ this may seem to imply that the correlator (15) is zero, bringing about the suspicion for the BRST triviality of $V_{5}$ However, a more careful look at the problem shows that this argument only implies the vanishing of some (but not all) of the correlation functions of $V_{5}$ but, strictly speaking, says nothing about its BRST triviality. That this argument does not imply the vanishing of all the correlators of $V_{5}$, can be shown from the following. The non-singlular OPE of $\Gamma$ with $U_{5}$ is given by

$$
\Gamma(z) U_{5}(w) \sim(z-w)^{2} e^{-2 \phi} \psi^{\left[m_{1}\right.} \ldots \psi^{m_{4}}\left(i(k \psi) \psi^{\left.m_{5}\right]}+\partial X_{\left.m_{5}\right]}\right) e^{i k X}
$$

(here we imply the antisymmetrization of over the space-time indices and ignore the $c \partial \xi$ term of $\Gamma$ which is irrelevant for integrated vertices). This operator product vanishes for $z=w$. Imagine, however, that the correlation function involving $U_{5}$ also contains another integrated physical operator, $\oint \frac{d u}{2 i \pi} W_{5}(u)$ such that the OPE of $\Gamma$ and $W_{5}$ is singular and the singularity order is greater, or equal to $(z-u)^{-2}$. Suppose such a singularity cannot be removed by the picture-changing so it is not the artifact of a picture choice (e.g. as this would be the case for a photon at picture +1$)$. Then integral over $u$ particularly includes the vicinity of $z$, the location of $U_{5}$. If $\lim _{u \rightarrow w} \Gamma(z) W_{5}(u) \sim(z-w)^{-n} ; n \geq 2$, moving the location of $\Gamma$ from $z$ to $w$ can no longer annihilate the correlation function and hence corresponding amplitude will be nonzero. Thus, the important conclusion we draw is that (14) does not imply the vanishing of all of the amplitudes of $V_{5}$ but only of those not containing the operators having singular OPE's with $\Gamma$. For example, all the correlators of the type $<V_{5}\left(z_{1}\right) \ldots V_{5}\left(z_{m}\right) Z_{1}\left(u_{1}\right) \ldots Z_{n}\left(u_{n}\right)>$ where $Z_{i}\left(u_{i}\right)$ are perturbative superstring vertices (e.g. a photon) must certainly vanish. However, this does not imply the triviality of $V_{5}$. Consider an operator

$$
W_{5}=H_{m_{1} \ldots m_{5}} \oint \frac{d z}{2 i \pi} e^{\phi} \psi^{m_{1}} \ldots \psi^{m_{5}} e^{i k X}(z)
$$


which is similar to the five-form (5) with $e^{-3 \phi}$ replaced by the operator $e^{\phi}$ of the identical conformal dimension $-\frac{3}{2}$. Again, although this operator isn't manifestly BRST-invariant, below we shall demonstrate how its invariance can be restored by adding the $b-c$ ghost dependent terms. Its OPE with $\Gamma$ is given by

$$
\Gamma(z) e^{\phi} \psi^{m_{1}} \ldots \psi^{m_{5}} e^{i k X}(w) \sim(z-w)^{-2} e^{2 \phi} \psi^{m_{1}} \ldots \psi^{m_{4}}\left(i(k \psi) \psi m_{5}+\partial X_{m_{5}}\right) e^{i k X}(w)+\ldots
$$

i.e. is precisely what we are looking for. So our goal now is to derive the correction terms restoring the BRST-invariance of $W_{5}$ and to demonstrate the nonzero correlator involving the $V_{5}$ and $W_{5}$ operators. This would be a sufficient proof of the non-triviality of both $V_{5}$ and $W_{5}$. We start with the BRST invariance restoration. The strategy is the following. Consider the BRST charge given by

$$
Q_{b r s t}=\oint \frac{d z}{2 i \pi}\left(c T-b c \partial c-\frac{1}{2} \gamma \psi_{m} \partial X^{m}-\frac{1}{4} \gamma^{2} b\right)
$$

Introduce an operator

$$
L(z)=-4 c e^{2 \chi-2 \phi} \equiv: \xi \Gamma^{-1}:
$$

, satisfying $\left\{Q_{b r s t}, L\right\}=1$ Consider a non-invariant operator $V$ satisfying $\left\{Q_{b r s t}, V\right\}=W$ for some $W$. Then, as $W$ is BRST-exact, clearly the transformation $V \rightarrow V_{\text {inv }}=V-L W$ restores BRST-invariance, provided that $V_{i n v}$ is not exact (the latter has of course to be checked separately). Applying this scheme for $W_{5}$ of (17), we have

$$
\left[Q_{b r s t}, W_{5}\right]=H_{m_{1} \ldots m_{5}}(k) \int \frac{d z}{2 i \pi} e^{2 \phi-\chi+i k X} R_{1}^{m_{1} \ldots m_{5}}(z)+b e^{3 \phi-2 \phi+i k X} R_{2}^{m_{1} \ldots m_{5}}(z)
$$

where

$$
\begin{array}{r}
R^{m_{1} \ldots m_{5}}(z)=-\frac{1}{2} \psi^{m_{1}} \ldots \psi^{m_{5}}(\psi \partial X)-\frac{1}{2} \psi^{\left[m_{1}\right.} . . \psi^{m_{4}}\left(\partial^{2} X^{\left.m_{5}\right]}+\partial X^{\left.m_{5}\right]}(\partial \phi-\partial \chi)\right) \\
-\frac{i}{2} \psi^{m_{1}} \ldots \psi^{m_{5}}(k \psi)(\partial \phi-\partial \chi)+(k \partial \psi)
\end{array}
$$

and

$$
R_{2}^{m_{1} \ldots m_{5}}(z)=-\frac{1}{4}(2 \partial \phi-2 \partial \chi-\partial \sigma) \psi^{m_{1}} \ldots \psi^{m_{5}}
$$

The next step is to cast this commutator as

$$
=\frac{1}{2} H_{m_{1} \ldots m_{5}}(k) \oint_{w} \frac{d z}{2 i \pi}(z-w)^{2} \partial_{z}^{2}\left(e^{2 \phi-\chi} R_{1}^{m_{1} \ldots m_{5}}+e^{3 \phi-2 \chi} R_{2}^{m_{1} \ldots m_{5}}(z)\right)
$$


where $\mathrm{w}$ is some worldsheet point; the expression (24) can obviously be brought to the form (21) by partial integration. The contour integral is taken around $w$; as the choice of $w$ is arbitrary, any dependence on it in shall disappear in the end in correlation functions. The next step is to insert the L-operator (20) inside the integral (24). Evaluating the OPE of $L$ with the integrand of (24) we obtain

$$
\begin{array}{r}
W_{5 i n v}(k, w)=H_{m_{1} \ldots m_{5}}(k)\left\{\oint \frac{d z}{2 i \pi} e^{\phi} \psi^{m_{1}} \ldots \psi^{m_{5}} e^{i k X}\right. \\
\left.-\frac{1}{2} \oint_{w} \frac{d z}{2 i \pi}(z-w)^{2}: L \partial_{z}^{2}\left(e^{2 \phi-\chi} R_{1}^{m_{1} \ldots m_{5}}+e^{3 \phi-2 \chi} R_{2}^{m_{1} \ldots m_{5}}(z)\right):\right\} \\
=H_{m_{1} \ldots m_{5}}(k)\left\{2 \oint \frac{d z}{2 i \pi} e^{\phi} \psi^{m_{1}} \ldots \psi^{m_{5}} e^{i k X}-2 \oint_{w} \frac{d z}{2 i \pi}(z-w)^{2} c e^{\chi} R_{1}^{m_{1} \ldots m_{5}}(k, z)\right\}
\end{array}
$$

This concludes the construction of the BRST-invariant 5 -form state at picture +1 . However one still has to check if $W_{5}$-operator is non-trivial. This is the legitimate concern since the BRST-invariant integrand of (25) is BRST-invariant and has conformal dimension 3 and it is well-known that invariant operators of conformal dimension other than 0 can always be expressed as BRST commutators. Indeed, the commutator of any operator of dimension $\mathrm{h}$ with the zero mode of $T$ satisfies

$$
\left[T_{0}, V_{h}\right]=h V_{h}
$$

where $T_{0}=\oint \frac{d z}{2 i \pi} z T(z)$. Since $T_{0}=\left\{Q_{b r s t}, b_{0}\right\}$, for any invariant $V_{h}$ one has

$$
V_{h}=\frac{1}{h}\left\{Q_{b r s t}, b_{0} V_{h}\right\}
$$

Acting on (25) with $b_{0}$ gives

$$
b_{0} W_{5 i n v}=\frac{2}{3} \oint_{w} \frac{d z}{2 i \pi}(z-w)^{3} e^{\chi} R_{1}^{m_{1} \ldots m_{5}}(k, z)
$$

accordingly $W_{5 i n v}$ can be represented as commutator

$$
W_{5 i n v}=\left\{Q_{b r s t}, \frac{1}{6} \oint_{w} \frac{d z}{2 i \pi}(z-w)^{3} \xi R_{1}^{m_{1} \ldots m_{5}}(k, z)\right\}
$$

i.e. it is the BRST commutator with the operator outside the small Hilbert space. For this reason, the operator $W_{5 i n v}$ is BRST nontrivial. The commutator (29) also ensures that the $W_{5 i n v}$ operator is in the small Hilbert space despite its manifest dependence on $\xi$ since $\left\{Q_{b r s t}, \xi\right\}$ is just the picture-changing operator. An example of non-zero correlation 
function is a 3-point correlator of 2 five-form states and one photon. As it is clear from the above, one five-form should be taken at a picture +1 and another at a picture -3 , as any 3-point correlator containing the same-picture five-forms would vanish due to (14). Technically, this means that a photon state appears in the operator product of two fiveforms of positive and negative pictures, but not in the OPE of the same picture five-forms. The non-zero correlator involves one unintegrated picture +1 photon:

$$
V_{p h}(q, z)=A_{m}(q): \Gamma\left\{c\left(\partial X^{m}+i(k \psi) \psi^{m}\right)+\gamma \psi^{m}\right\} e^{i q X}:(z)
$$

one unintegrated picture -3 five-form:

$$
V_{5}(k, z)=H_{m_{1} \ldots m_{5}}(k): c e^{-3 \phi} \psi^{m_{1}} \ldots \psi^{m_{5}} e^{i k X}:(z)
$$

and one integrated five-form $W_{5 i n v}(p)$ of $(25)$ at picture +1 . Such a combination of pictures ensures the correct ghost number balance of the correlator necessary to cancel the background ghost charges - that is, the $\phi$ ghost number $-2, \chi$ ghostnumber +1 and $b-c$ ghost number -3 , due to the contribution of the correction term $\sim c \xi R_{1}^{m_{1} \ldots m_{5}}(k, z)$ of $W_{5}$. Such a three-point function is in some way unusual as the usual perturbative three-point correlators in string theory normally involve the unintegrated vertices only. Evaluating the correlator and evaluating the contour integral around the insertion point $z_{2}$ of $W_{5 i n v}$ we find the result to given by The result of the computation is given by

$$
<V_{5}\left(k, z_{1}\right) W_{5 i n v}\left(p, z_{2}\right) V_{p h}\left(q, z_{3}\right)>=H_{m_{1} \ldots m_{5}}(k) H^{m_{1} \ldots m_{5}}(p)((k q)(p A)-(p q)(k A))
$$

After the Fourier transform, this correlator leads to the low-energy effective action term given by

$$
S_{e f f} \sim \int d^{d} X(d H)_{l_{1} \ldots l_{5}}^{m}(d H)^{l_{1} \ldots l_{5} n} F_{m n}
$$

Remarkably, this term, originating from the worldsheet correlator of two five-forms with a photon on a sphere has the structure identical to the one obtained from the disc amplitude of a photon with two Ramond-Ramond operators of the 6 -form field strengths $d H$. Thus the $V_{5}$ open string five-form state can be interpreted as a source of the Ramond-Ramond 5-form charge, i.e. a D4-brane. This calculation also illustrates the BRST non-triviality of $V_{5}$ and $W_{5}$ states. Having illustrated the appearance of the picture-dependent physical vertex operators, we are now prepared to give a formal definition of ghost cohomologies. 
1)The positive ghost number $N$ cohomology $H_{N}(N>0)$ is the set of physical (BRST invariant and non-trivial) vertex operators that exist at positive superconformal ghost pictures $n \geq N$ and that are annihilated by the inverse picture-changing operator $\Gamma^{-1}=$ $-4 c \partial \xi e^{-2 \phi}$ at the picture $N .: \Gamma^{-1} V^{(N)}:=0$ This means that the picture $N$ is the minimum positive picture at which the operators $V \subset H_{N}$ can exist.

2)The negative ghost number $-N$ cohomology $H_{-N}$ consists of the physical vertex operators that exist at negative superconformal pictures $n \leq-N(N>0)$ and that are annihilated by the direct picture changing at maximum negative picture $-N:: \Gamma V^{(-N)}:=$ 0 .

3)The operators existing at all pictures, including picture zero, at which they decouple from superconformal ghosts, are by definition the elements of the zero ghost cohomology $H_{0}$. The standard string perturbation theory thus involves the elements of $H_{0}$, such as a photon. The picture -3 and picture +1 five-forms considered above are the elements of $H_{-3}$ and $H_{1}$ respectively.

4) Generically, there is an isomorphism between the positive and the negative ghost cohomologies: $H_{-N-2} \sim H_{N} ; N \geq 1$, as the conformal dimensions of the operators $: e^{-(N+2) \phi}(z)$ and $e^{N \phi}$ are equal and given by $-\frac{N^{2}}{2}-N$. That is, to any element of $H_{-N-2}$ there corresponds an element from $H_{N}$, obtained by replacing $e^{-(N+2) \phi} \rightarrow e^{N \phi}$ and adding the appropriate $b-c$ ghost terms in order to restore the BRST-invariance, using the Loperator (20). For this reason, we shall refer to the cohomologies $H_{-N-2}$ and $H_{N}$ as dual. With some effort, one can also work out the precise isomorphism relation between $H_{N}$ and $H_{-N-2}$ That is, let the integrated vertex operator $h_{-N-2} \equiv \oint \frac{d z}{2 i \pi} W_{-N-2}(z) \subset H_{-N-2}$ be the element of $H_{-N-2}$, with the integrand $W_{-N-2}(z)$ being some dimension 1 operator (for simplicity, we take it at tis maximal negative picture $-N-2$ ). The unintegrated version of this operator is given by $c W_{-N-2}$ Then the corresponding operator, the element of $H_{N}: h_{N} \equiv \oint \frac{d z}{2 i \pi} W_{N}(z) \subset H_{N}$ can be constructed as

$$
h_{N}=: \Gamma:^{2 N+2}: Z c W_{-N-2}:
$$

where

$$
Z(u)=\oint \frac{d w}{2 i \pi}(u-w)^{3}\left(b T+4 c \xi \partial \xi e^{-2 \phi} T^{2}\right)(w)
$$

(with $T$ being the full matter+ghost stress-energy tensor of the RNS theory) is non-local, BRST-invariant and non-trivial $Z$-operator mapping unintegrated vertices into integrated ones. The properties of this operator have been discussed in [2] and basically, it can 
be viewed as a $b-c$ analogue of $\Gamma$ (from this point of view, integrated and unintegrated vertices are just two versions of the same operator at different $b-c$ pictures). Since $\Gamma$ is invariant and non-trivial, $h_{N}$ is physical by construction, once $h_{-N-2}$ is invariant and non-trivial. The $L$-operator prescription for the five-form, described above, is simply one explicit example of building such an isomorphism. Technically, it is the non-local structure of $Z$ that leads to the terms from higher Fourier modes in the expression (25) for $W_{5 i n v}$, though such terms usually don't appear in expressions for standard vertex operators, such as a photon. Strictly speaking, the isomorphism between $H_{N}$ and $H_{-N-2}$ cannot be reduced to a usual picture equivalence (though it is very much reminiscent of the one) since the operators $Z$ and $\Gamma$ do not commute. Note that $H_{-1}$ and $H_{-2}$ are empty, as any operator existing at pictures -2 or -1 is either trivial or can always be brought to picture zero by $\Gamma$.

The important property of the ghost cohomologies is that typically, the elements of

$H_{-N}$ have singular operator products with the inverse picture-changing $\Gamma^{-1}$ while the elements of $H_{N}$ have singular OPE's with the direct picture-changing $\Gamma$. As has been explained above, it is this property that ensures that correlators containing the vertices annihilated by direct and inverse picture-changing, do not vanish despite (14), provided that the correlator contains at least two vertices from the dual cohomologies of opposite signs. At least one of these vertices must be taken in the integrated form, for the reason we have pointed out above. In critical strings, the only nontrivial cohomologies are $H_{-3}$ and its dual $H_{1}$, i.e. the five-form states (5), (25). In non-critical strings, because of the Liouville dressing, cohomologies of higher ghost numbers may appear as well. In the next section we will show that the dimension 1 currents from the higher ghost cohomologies enhance the symmetry algebra of the target space and thus can be regarded as the generators of hidden space-time symmetries originating from extra dimensions.

\section{Ghost cohomologies, hidden symmetries and 2T-physics}

The properties of the picture-dependent five-forms (5),(25) and their closed string counterparts have been studied in a number of papers in the past (see e.g. [4], [3], [5]) In particular, we have been able to show the relevance to the non-perturbative dynamics of $D$-branes. For example, the picture-dependent operators of the closed string sector can be understood as the creadtion operators for $D$-branes in the second quantized formalism [3]. This particularly means that the picture-dependent vertices of the type (5), (25) (more precisely, their closed string counterparts) describe the non-perturbative processes like an emission of a $D$-brane by a string. Of course, once such a $D$-brane has been created "ex 
nihilo", this would lead to strong deformations of the space-time geometry. For instance, if an emission took place in the originally flat space-time, the D-brane appearance in the vacuum would result in strong fluctuations of space-time metric which would gradually stabilize to the appropriate D brane-type configuration. The processes of this type, describing the evolution of the space-time metric from flat to curved D-brane geometry, have been studied in the past by analyzing the worldsheet RG equations for sigma-models with the picture-dependent operators, and it has been realised that generally these metric fluctuations are described by either the stochastic equations of the Langevin type with the non-Markovian noise or, in more complicated cases, the Navier-Stokes type equations of hydrodynamics [5]. In this context, the flat and the curved D-brane metric can be viewed as different thermodynamical limits of the above stochastic processes. Although it has been understood that the appearance of picture-dependent vertices strongly reshapes the geometry of space-time, certain general principle, relating the picture-dependent states to deformations of geometry (including the possible appearance of new space-time symmetries and extra dimensions) has been lacking so far. In this section we shall attempt to formulate such a principle by studying the example of the ghost cohomologies in noncritical NSR string theories with the Liouville dressing. The particular result that we shall demonstrate below is the surprising relation between the ghost cohomologies and the extra symmetries from hidden space-time dimensions, observed in Bars $2 \mathrm{~T}$ theories. For example, one particular observation made by Bars is that the full symmetry group of a particle in the $A d S_{d}$ space is given by $S O(d, 2)$ which is larger than the naive $S O(d-1,2)$ isometry group of the $A d S_{d}$ space [6]. It has been shown by Bars that the generators of these extra symmetries originate from hidden dimensions, one of which is time-like [6], [7]. In this section, we shall discuss the string-theoretic analogue of these new symmetries. Namely, we will show that the off-shell transformations in the $2 \mathrm{~T}$ theories observed by Bars et.al [6], [7], [8], leading to non-linear symmetry transformations with their origin in hidden space-time dimensions, are produced by generators isomorphic to the picture-dependent currents of higher ghost cohomologies. Moreover, we shall argue that the off-shell symmetries and extra dimensions, observed by Bars [6], [7], [8] may not be complete as they are isomorphic to operators of the lowest nonzero ghost cohomologies; thus "switching on" the cohomologies of higher ghost numbers may result in the extra symmetries not yet observed. The main idea behind the relation of ghost cohomologies to extra space-time symmetries is quite simple. In superstring theory, the generators of space-time symmetries are given by the worldsheet integrals of primary fields of dimension 1. For example, in flat 
space-time the dimension 1 operators $T^{m}=\oint \frac{d z}{2 i \pi} \partial X^{m}, L^{m n}=\oint \frac{d z}{2 i \pi} \psi^{m} \psi^{n} ; m=0, \ldots d-1$ generate $d$ translations and $\frac{1}{2}(d-1) d$ rotations of the Poincare group. These generators can of course be taken at equivalent ghost pictures; for instance at picture -1 we have $T^{m}=\oint \frac{d z}{2 i \pi} e^{-\phi} \psi^{m} ; L^{m n}=\oint \frac{d z}{2 i \pi} c \partial \xi e^{-2 \phi} \psi^{m} \psi^{n}$. The current algebra of these operators generates the space-time symmetry group and the manifest space-time symmetries are therefore in one to one correspondence with the dimension one primary operators of ghost number zero cohomology with zero momenta. The appearance of the primary dimension 1 fields from cohomologies of nonzero ghost numbers, however, extends the current algebra and thus leads to new transformations in space-time, which are the candidates for new space-time symmetries (the latter should be checked separately, upon the construction of the generators). We shall demonstrate that these extra generators correspond to the hidden space-time symmetries, observed by Bars. As an example, we will consider the noncritical superstrings in $d-1$ dimensional Minkowski space-time $(d \geq 1)$. It is easy to check that in this theory the dimension 1 primary fields (including the matter and the Liouville coordinates) with momentum zero in the $X$-direction generate $S O(d-1,2)$ current algebra, which is identical to the isometry of critical strings in $A d S_{d}$. That is, let $\varphi$ be the Liouville field and $\lambda$ its worldsheet superpartner. Then it's easy to check that the dimension 1 primaries generating $S O(d-1,2)$ are given by (in the limit of zero cosmological constant):

$$
\begin{aligned}
& L^{m n}=\oint \frac{d z}{2 i \pi} \psi^{m} \psi^{n} \\
& L^{+m}=\oint \frac{d z}{2 i \pi} e^{-\phi} \psi^{m} \\
& L^{-m}=l(d) \oint \frac{d z}{2 i \pi} e^{Q \varphi} \psi^{m} \lambda \\
& L^{+-}=l(d) \oint \frac{d z}{2 i \pi} e^{-\phi+Q \varphi} \lambda
\end{aligned}
$$

where $l(d)$ are the normalization constants for the Liouville-dependent operators, needed to compensate for the finite renormalization of the structure constants due to the Liouville dressing [9] (see the discussion below). The stress-energy tensor and the supercurrent for the super Liouville system are given by

$$
\begin{array}{r}
T_{\varphi, \lambda}=-\frac{1}{2}(\partial \varphi)^{2}+\frac{Q}{2} \partial^{2} \varphi-\frac{1}{2} \partial \lambda \lambda \\
G_{\varphi, \lambda}=-\frac{1}{\sqrt{2}}(\lambda \partial \varphi+Q \partial \lambda)
\end{array}
$$


and the value of the background charge in $d$ dimensions is given by

$$
Q=\sqrt{\frac{9-d}{2}}
$$

is chosen so that the total central charge of the system is zero:

$$
c_{X}+c_{\psi}+c_{\varphi}+c_{\lambda}+c_{b-c}+c_{\beta \gamma}=0
$$

and thus the conformal dimension of $e^{Q \varphi}$ is zero. Next, $\eta^{m n}$ is the Minkowski metric, while $\eta^{+-}=-1 ; \eta^{++}=\eta^{--}=0$.

The dressing with the dimension zero $e^{Q \varphi}$ operator is necessary in order to ensure that the Liouville dependent currents are the primary fields, due to the second derivative term in the Liouville stress tensor. Evaluating the commutators of the Liouville-dependent generators, one has to compute their OPE's without the dressing first, and then to dress the obtained operator on the right-hand side, following the procedure similar to [9]. As a result of the dressing, the structure constants are renormalized by the factor of $\frac{k(d)+2}{k(d)+1}$ [9] where $k(d)$ is the central charge of $S L(2, R)$ current algebra in super Liouville theory given by [9]:

$$
k(d)=\frac{1}{8}(d-1 \pm \sqrt{(d-1)(d-9)})
$$

Accordingly, the normalization constants $l(d)$ of $(34)$ are to be chosen as

$$
l(d)=\sqrt{\frac{k(d)+1}{k(d)+2}}
$$

Our goal now is to demonstrate that for all values of $d$ (except for the special cases of the $c=1$ theory with $d-1=1$ and critical superstrings with $d-1=10$ ) the picturedependent dimension 1 primaries from $H_{1} \sim H_{-3}$ are given by $d+1$ generators, extending the $S O(d-1,2) A d S_{d}$ isometry to $S O(d, 2)$. Such an enhancement of the space-time symmetry group is precisely the one observed by Bars [6] for a particle in $A d S_{d}$ due to $d+1$ extra generators which, in the interpretation of [6] originate from hidden space-time dimensions of the $2 \mathrm{~T}$-theory living in $d+2$ dimensions, in which the $d$-dimensional universe is embedded. As in [6], these extra operators generate the nonlinear transformations of the worldsheet Lagrangian shifting it by a total derivative, i.e. leaving the action invariant. In this context, the two extra dimensions observed by Bars admit a simple interpretation: one of them is the Liouville field, another is the cohomology of the lowest nontrivial number. As 
we have mentioned before, it is natural to assume that the higher dimensional cohomologies lead to further non-linear space-time symmetries, so far unobserved in the $2 \mathrm{~T}$ physics formalism. We start from reviewing the case of $d=2$ (supersymmetric $c=1$ model) and then generalise it for higher values of $d$. The case of $c=1$ model is special since, even without the operators from nonzero ghost cohomologies, it contains $3 S U(2)$ space-time generators, well-known to generate the set of $S U(2)$ multiplet discrete states [10], [11], [12]:

$$
\begin{array}{r}
T_{0,0}=\oint \frac{d z}{2 i \pi} \partial X \\
T_{0,1}=\oint \frac{d z}{2 i \pi} e^{i X} \psi \\
T_{0,-1}=\oint \frac{d z}{2 i \pi} e^{-i X} \psi
\end{array}
$$

where the left index refers to the ghost number and the right to the integer momentum in the $X$-direction. It has been shown [13] that the currents from $H_{1} \sim H_{-3}$ extend the $S U(2)$ current algebra to $S U(3)$. Five extra generators from $H_{1} \sim H_{-3}$ are given by [13]:

$$
\begin{array}{r}
T_{-3,2}=\oint \frac{d z}{2 i \pi} e^{-3 \phi+2 i X} \psi(z) \\
T_{-3,1}=\oint \frac{d z}{2 i \pi} e^{-3 \phi+i X}\left(\partial \psi \psi+\frac{1}{2}(\partial X)^{2}+\frac{i}{2} \partial^{2} X\right)(z) \\
T_{-3,-1}=\oint \frac{d z}{2 i \pi} e^{-3 \phi-i X}\left(\partial \psi \psi+\frac{1}{2}(\partial X)^{2}-\frac{i}{2} \partial^{2} X\right)(z) \\
T_{-3,0}=\oint \frac{d z}{2 i \pi} e^{-3 \phi}\left(\partial^{2} X \psi-2 \partial X \partial \psi\right)(z) \\
T_{-3,-2}=\oint \frac{d z}{2 i \pi} e^{-3 \phi-2 i X} \psi(z)
\end{array}
$$

It is straightforward to check that these operators are the BRST-invariant and non-trivial primary fields of dimension one, annihilated by $\Gamma$ and are thus the elements of $H_{-3}$. Of course, the $H_{1}$-version of these operators can be constructed as well by replacing $e^{-3 \phi}$ with $e^{\phi}$ and adding the $b-c$ ghost terms by using the L-operator prescription (20).

The $S U(3)$ algebra is then generated by combining (41) with 3 operators (40) of $S U(2)$ The Cartan subalgebra of $S U(3)$ is formed by the zero momentum generators $T_{0,0}$ and $T_{-3,0}$ while 3 operators with negative and 3 with positive momenta form the lower and upper nilpotent subalgebras respectively. The SU(3) multiplet states are then obtained by acting with various combinations of the lowering operators $T_{-3,-1}, T_{-3,-2}$ and 
$T_{0,-1}$ on the dressed tachyonic highest weight vectors with positive integer momenta. The prescription to obtain these operators is the following: one starts with maximal positive momentum generator $T_{-3,2}$ of $H_{-3}$ and acts on it repeatedly with the lowering $T_{0,-1}$ of $S U(2)$, descending down to the maximal negative momentum generator $T_{-3,-2}$ (which in turn is annihilated by $\left.T_{0,-1}\right)$ thus generating 5 currents $T_{-3, n}$ of $H_{-3}(-2 \leq n \leq 2)$. The described procedure is straightforward to generalize for any higher ghost number hohomology $H_{-n} \sim H_{n-2} ; n \geq 3$. That is, one starts with the maximum positive momentum $H_{-n}$ primary $T_{-n, n-1}=\oint \frac{d z}{2 i \pi} e^{-n \phi+i(n-1) X}$ and then generates $n+1 T_{-n, m}$ generators of $H_{-n}$ or its dual $H_{n-2}(-n \leq m \leq n)$ by repeated action on $T_{-n, n-1}$ with $T_{0,-1}$. Then, combining together all the operators from $H_{-i} \sim H_{i-2} ; i=0,3,4, \ldots, n$ one obtains $n^{2}-1$ currents generating $S U(n)$, although the explicit expressions for the generators become quite cumbersome for $n>3$ [13]. Again, the $S U(n)$ multiplet states are obtained by acting on the dressed tachyonic primaries with integer positive momenta with various combinations of $T_{-n, m} \sim T_{n-2, m}$ with $m<0$. Their structure constants are given by the Clebsch-Gordan coefficients of $S U(n) \sim S L(n, R)$ (up to the factors - functions of the Casimir eigenvalues) and are thus related to the volume preserving diffeomorphisms in $N$ dimensions SDiff $(n)$. Remarkably, this implies that each time we "switch on" the generators from a cohomology of the higher ghost number, we "open up" an extra space-time dimension.

Let us now consider the case of non-critical strings in higher dimensions. The direct generalization of the procedure, described for the case of $c=1$, doesn't work for $d-1>$ 1 , as we no longer have generators with the discrete nonzero momenta. For example, the analogue of the current $T_{-n, n-1}$ is now the operator $\oint \frac{d z}{2 i \pi} e^{-n \phi+i k_{m} X^{m}}$ where the momentum $\vec{k}$ satifies $k_{m} k^{m}=n-1$, but is arbitrary otherwise. For this reason, the only $H_{-n} \sim H_{n-2}$-operators $(n \neq 0,1,2)$ that the higher dimensional strings could "inherit" from the $c=1$ case are those carrying momentum zero. That is, they should inherit the structure of the Cartan generators of $S U(n)$. Consider the case $n=3$. The straightforward analogue of the $T_{-3,0}$ Cartan generator of $S U(3)$ is the trace operator

$$
L^{+\alpha}=\oint \frac{d z}{2 i \pi} e^{-3 \phi}\left(\partial^{2} X_{m} \psi^{m}-2 \partial X_{m} \partial \psi^{m}\right)
$$

where by definition $\alpha=1, \eta^{\alpha \alpha}=1$.

The Greek space index $\alpha$ corresponds to what will turn out to be an extra dimension, induced by the generators of $H_{-3} \sim H_{1}$; of course, it should be distinguished from $m=1$ of the $(d-1)$-dimensional Minkowski space. Note that the generators

$$
\rho_{m}^{n}=\oint \frac{d z}{2 i \pi} e^{-3 \phi}\left(\partial^{2} X_{m} \psi^{n}-2 \partial X_{m} \partial \psi^{n}\right)
$$


are not the elements of $H_{-3}$ for $n \neq m$ since they are BRST-exact (except for $n=m$ ). That is, it is straightforward to check that

$$
\rho_{m}^{n}=\left[Q_{b r s t},: \Gamma^{-1} U_{m}^{n}:\right]
$$

with

$$
U_{m}^{n}=\oint \frac{d z}{2 i \pi} \partial \xi e^{-3 \phi} \psi_{m} \partial X^{n}
$$

On the other hand,the generator $L^{+\alpha} \equiv \operatorname{Tr} \rho_{m}^{n}$ is nontrivial as for the coincident $m$ and $n\left[Q_{b r s t}, U_{m}^{m}\right]=0$. Next, using the Liouville field $\varphi$ and its worldsheet superpartner $\lambda$ it is not difficult to construct other $d H_{-3}$-generators of the $T_{-3,0}$-type. That is, it is straightforward to check that $d-1$ generators

$$
\begin{array}{r}
L^{m \alpha}=l(d) \oint \frac{d z}{2 i \pi} e^{-3 \phi+Q \varphi}\left\{\left(\partial^{2} \varphi+Q(\partial \varphi)^{2}\right) \psi^{m}-2 \partial \varphi \partial \psi^{m}+\partial^{2} X^{m} \lambda\right. \\
\left.-2 \partial X^{m} \partial \lambda-4 Q \partial \varphi \lambda \partial X^{m}\right\}
\end{array}
$$

are the BRST-invariant and non-trivial primary fields of dimension 1, annihilated by $\Gamma$, i.e. are the elements of $H_{-3} \sim H_{1}$ The final generator of the $T_{-3,0}$-type, the element of $H_{-3} \sim H_{1}$ can be constructed using just the superconformal ghosts, $\varphi$ and $\lambda$. It is given by

$$
L^{-\alpha}=l(d) \oint \frac{d z}{2 i \pi} e^{\phi+Q \varphi}\left\{\left(\partial^{2} \varphi+Q(\partial \varphi)^{2}\right) \lambda+\partial \varphi \partial \lambda\right\}
$$

The final step is to check that $d+1$ generators $L^{+\alpha}, L^{-\alpha}$ and $L^{m \alpha}$ of $H_{-3}$, along with $\frac{1}{2}(d-1) d$ generators of $S O(d-1,2)$ combine into $\frac{1}{2} d(d+1)$ generators of $S O(d, 2)$

Introducing the $d+2$-dimensional index $M=(m,+,-, \alpha) ; m=0, \ldots d-2 ; \alpha=1$ with the $(d, 2)$ metric $\eta^{M N}$ consisting of $\eta^{m n}, \eta^{+-}=-1, \eta^{--}=\eta++=0, \eta^{\alpha \alpha}=1$ and evaluating the commutators one can show that

$$
\left[L^{M_{1} N_{1}}, L^{M_{2} N_{2}}\right]=\eta^{M_{1} M_{2}} L^{N_{1} N_{2}}+\eta^{N_{1} N_{2}} L^{M_{1} M_{2}}-\eta^{M_{1} N_{2}} L^{M_{2} N_{1}}-\eta^{M_{2} N_{1}} L^{M_{1} N_{2}}
$$

As before, the commutators of the operators (46), (47) must first be computed in the limit of zero dilaton field (i.e. with the generators taken without the dressing) with the subsequent Liouville dressing of the r.h.s. of the obtained commutator. In the commutators (48) with the both of $L^{M N}$ 's coming from $H_{-3} \sim H_{1}$ one has to take one of the generators at picture -3 and another at picture +1 , since only the OPE's of the currents from the dual cohomologies of the opposite sign $\left(H_{1}\right.$ and $\left.H_{-3}\right)$ contain the non-trivial operators (typically, those of $\left.H_{0}\right)$. On the contrary, the OPE of any 2 operators which are both from $H_{-3}$ or 
both from $H_{1}$ cannot contain any non-trivial currents of $H_{0}$, as has been explained above. Structurally, the commutator of two currents from $H_{-3}$ and $H_{1}$ gives the current from $H_{0}$ at picture -2 . The commutators of currents from $H_{-3}$ (or $H_{1}$ ) with operators from $H_{0}$ typically produce the currents of $H_{-3}\left(\right.$ or $\left.H_{1}\right)$. The $L^{M N}$-commutators constructed above are thus in one to one correspondence with the L-generators of $S O(d, 2)$, observed by Bars for the $A d S_{d}$ particle. The generators from the nonzero ghost cohomologies are in one to one correspondence with the generators from hidden extra dimensions in Bars approach.

\section{T-physics and $\alpha$-symmetry}

To conclude this paper, we will demonstrate that the generators $L^{-\alpha}, L^{+\alpha}$ and $L^{m \alpha}$ of $L^{M N}$ generate the nonlinear transformations of the worldsheet $N S R$ Lagrangian that leave it invariant, up to total derivative. These extra symmetries are similar to the non-linear symmetry transformations for $A d S_{d}$ particle, first observed by Bars. We start with the symmetry transformations generated by the trace operator $L^{+\alpha}$. Applying the generator $\epsilon^{+\alpha} L^{+\alpha}$ to various $R N S$ fields (where $\epsilon^{+\alpha}$ is the infinitezimal parameter) one easily finds the corresponding transformations to be given by

$$
\begin{array}{r}
\delta X^{m}=\epsilon^{+\alpha}\left\{\partial\left(e^{\phi} \psi^{m}\right)+2 e^{\phi} \partial \psi^{m}\right\} \\
\delta \psi^{m}=\epsilon^{+\alpha}\left\{-e^{\phi} \partial^{2} X^{m}-2 \partial\left(e^{\phi} \partial X^{m}\right)\right\} \\
\delta \gamma=\epsilon^{+\alpha} e^{2 \phi-\chi}\left(\psi_{m} \partial^{2} X^{m}-2 \partial \psi_{m} \partial X^{m}\right) \\
\delta \beta=\delta b=\delta c=0
\end{array}
$$

and similarly for the anti-holomorphic fields.

The next step is to show that the RNS worldsheet action:

$$
\begin{array}{r}
S_{N S R}=\frac{1}{2 \pi} \int d^{2} z\left\{-\frac{1}{2} \partial X_{m} \bar{\partial} X^{m}-\frac{1}{2} \psi^{m} \bar{\partial} \psi^{m}-\frac{1}{2} \bar{\psi}_{m} \partial \bar{\psi}^{m}\right. \\
+b \bar{\partial} c+\bar{b} \partial \bar{c}+\beta \bar{\partial} \gamma+\bar{\beta} \partial \bar{\gamma}\}+S_{\text {Liouville }} \\
S_{\text {Liouville }}=\frac{1}{4 \pi} \int d^{2} z\left\{\partial \varphi \bar{\partial} \varphi+\lambda \bar{\partial} \lambda+\bar{\lambda} \partial \bar{\lambda}-F^{2}+2 \mu_{0} b e^{b \varphi}(i b \lambda \bar{\lambda}-F)\right\}
\end{array}
$$

with

$$
Q=b+\frac{1}{b}
$$

is invariant under the transformations (49). Integrating by parts, we have: 


$$
\begin{array}{r}
\delta\left(-\frac{1}{4 \pi} \int d^{2} z \partial X_{m} \bar{\partial} X^{m}\right)=\frac{1}{2 \pi} \int d^{2} z \delta X_{m} \partial \bar{\partial} X^{m} \\
=\frac{1}{2 \pi} \int d^{2} z\left\{\partial\left(e^{\phi} \psi_{m}\right)+2 e^{\phi} \partial \psi_{m}\right\} \partial \bar{\partial} X^{m} \\
=\frac{1}{2 \pi} \int d^{2} z\left\{-e^{\phi} \psi_{m} \partial^{2} \bar{\partial} X^{m}+2 e^{\phi} \partial \psi_{m} \partial \bar{\partial} X^{m}\right\}
\end{array}
$$

Next, the transformation of the holomorphic $\psi_{m} \bar{\partial} \psi^{m}$-term of the worldsheet action (50) under (49) is given by:

$$
\begin{array}{r}
\delta\left(-\frac{1}{4 \pi} \int d^{2} z \psi_{m} \bar{\partial} \psi^{m}\right)=\frac{1}{2 \pi} \int d^{2} z \bar{\partial}\left(\delta \psi_{m}\right) \psi^{m} \\
=-\frac{1}{2 \pi} \int d^{2} z \bar{\partial}\left\{e^{\phi} \partial^{2} X_{m}+2 \partial\left(e^{\phi} \partial X_{m}\right)\right\} \psi^{m} \\
=-\frac{1}{2 \pi} \int d^{2} z\left\{\bar{\partial}\left(e^{\phi} \partial^{2} X_{m}\right) \psi^{m}-2 \bar{\partial}\left(e^{\phi} \partial X_{m}\right) \partial \psi^{m}\right\}
\end{array}
$$

Adding these contributions together, we get

$$
\begin{array}{r}
\delta S_{X}+\delta S_{\psi} \equiv \delta\left(-\frac{1}{4 \pi} \int d^{2} z\left\{\partial X_{m} \bar{\partial} X^{m}+\psi_{m} \bar{\partial} \psi^{m}\right\}\right) \\
=-\frac{1}{2 \pi} \int d^{2} z\left(\bar{\partial} e^{\phi}\right)\left\{\partial^{2} X_{m} \psi^{m}-2 \partial X_{m} \partial \psi^{m}\right\}
\end{array}
$$

Finally, using the OPE relation

$$
\lim _{z \rightarrow w}: \beta:(z): e^{2 \phi-\chi}:(w) \equiv \lim _{z \rightarrow w}: e^{\chi-\phi} \partial \chi:(z): e^{2 \phi-\chi}(w):=-: e^{\phi}:(z)
$$

and the fact that $\delta \beta=0$ we find the transformation of the $\beta-\gamma$ term of $S_{R N S}$ to be given by

$$
\begin{array}{r}
\left.\delta S_{\beta \gamma} \equiv \delta\left(\frac{1}{2 \pi} \int d^{2} z \beta \bar{\partial} \gamma\right)=-\frac{1}{2 \pi} \int d^{2} z \bar{\partial} \beta \overline{(} \delta \gamma\right) \\
=\frac{1}{2 \pi} \int d^{2} z\left(\bar{\partial} e^{\phi}\right)\left\{\partial^{2} X_{m} \psi^{m}-2 \partial X_{m} \partial \psi^{m}\right\}
\end{array}
$$

i.e.

$$
\delta\left(S_{X}+S_{\psi}+S_{\beta \gamma}\right)=0
$$

Since the $b-c$ and the Liouville parts of the RNS action (50) are manifestly invariant under (49), this means that $\delta S_{R N S}=0$ and the transformations (49) constitute the new space-time symmetry non-critical $R N S$ superstring theories. We shall refer to this new nonlinear symmetry of $S_{R N S}$ as the $\alpha$-symmetry and the corresponding transformations 
(49) as $\alpha$-transformations, in order to emphasize their relation to nonzero ghost cohomology generator $L^{+\alpha} \subset H_{-3} \sim H_{1}$ of $S O(d, 2)$ and, accordingly, to the hidden space-time dimension, which we have parametrized with the index $\alpha$ (see above). In case of the critical ten-dimensional superstring theory this is the only new additional symmetry of the RNS action (apart from the usual translations and rotations). For non-critical RNS strings in $d-1$ dimensions, however, there are also $d$ additional $\alpha$-transformations, generated by the Liouville-dependent $L^{-\alpha}$ and $L^{m \alpha}$ of $S O(d, 2)(m=0, \ldots, d-2)$. Proceeding exactly as above, one can easily derive $d-1 \alpha$-symmetry transformations for $S_{R N S}$, generated by $\epsilon_{m \alpha} L^{m \alpha}$, to be given by, in the zero cosmological constant limit and in the absense of dilaton:

$$
\begin{array}{r}
\delta X_{m}=\epsilon_{m \alpha}\left\{\partial\left(e^{\phi} \lambda\right)+2 e^{\phi} \partial \lambda\right\} \\
\delta \lambda=-\epsilon_{m \alpha}\left\{2 \partial\left(e^{\phi} \partial X^{m}\right)+e^{\phi} \partial^{2} X^{m}\right\} \\
\delta \gamma=\epsilon_{m \alpha} e^{2 \phi-\chi}\left\{\partial^{2} X^{m} \lambda-2 \partial X^{m} \partial \lambda\right\} \\
\delta \beta=\delta b=\delta c=\delta \varphi=\delta \psi^{m}=0
\end{array}
$$

The final, $(\mathrm{d}+1)$-st $\alpha$-symmetry transformation of $S_{N S R}$, generated by $L^{-\alpha}$ of (47) acts on the superconformal ghosts and the Liouville field only and is given by

$$
\begin{array}{r}
\delta \varphi=\epsilon_{-\alpha}\left\{\partial\left(e^{\phi} \lambda\right)+2 e^{\phi} \partial \lambda\right\} \\
\delta \lambda=-\epsilon_{-\alpha}\left\{2 \partial\left(e^{\phi} \partial \varphi\right)+e^{\phi} \partial^{2} \varphi\right\} \\
\delta \gamma=\epsilon_{-\alpha} e^{2 \phi-\chi}\left\{\lambda \partial^{2} \varphi-2 \partial \varphi \partial \lambda\right\} \\
\delta \beta=\delta b=\delta c=\delta X^{m}=\delta \psi^{m}=0
\end{array}
$$

Repeating the derivation we have performed above for $L^{+\alpha}$, it is now easy to check that the $\alpha$-transformations (57), (58) are the symmetries of the noncritical RNS. Thus the full $S O(d, 2)$ symmetry group of non-critical strings in $d-1$ consists of $\frac{1}{2} d(d-1)$ transformations of $A d S_{d}$ isometry plus $d+1 \alpha$-transformations generated by currents from nonzero ghost cohomology $H_{-3} \sim H_{1}$. This non-linear $\alpha$-symmetry is thus the new matter-ghost mixing symmetry of the RNS strings and must be the stringy analogue of the non-linear symmetries for the $A d S_{d}$ particle from hidden extra dimensions, previously observed in the $2 T$-physics approach.

\section{Discussion}

In this paper we have demonstrated that the appearance of the new physical ghostdependent generators from the first non-trivial ghost cohomology $H_{-3} \sim H_{1}$ in non-critical RNS superstring theories leads to the enhancement of the current algebra of space-time 
generators from $S O(d-1,2)$ to $S O(d, 2)$ and to new non-trivial global symmetries in space-time, associated with the new ghost-dependent generators. The non-linear symmetry transformations, called $\alpha$-transformations in this paper, are the symmetries of the $R N S$ Lagrangian. The generators of the $\alpha$-symmetry appear to be in one to one correspondence with the extra symmetry generators of the $2 T$ theories which, in the Bars approach, are related to the hidden symmetries originating from higher space-time dimensions. So in this sense, our results are the stringy extention of the work by Bars. The $\alpha$-symmetry of the worldsheet superstring action, discussed in this paper, has not been previously observed and this by itself appears to be an important finding. The natural question is what happens if one considers the ghost cohomologies of higher ghost numbers, for example, $H_{2} \sim H_{-4}$. In the special, and the simplest case of supersymmetric $c=1$ theory, each new cohomology effectively corresponds to opening up a theory to a new hidden space-time dimension. From the technical point of view, the $c>1$ case appears to be more complicated, but it is tempting to assume that qualitatively the same scenario is still true for $c>1$ : that is, the extra space-time generators from $H_{n} \sim H_{-n-2} ; n=$ $2,3, \ldots$ correspond to yet unknown space-time symmetries of the theory, related to yet unknown hidden space-time dimensions. Technically, the space-time symmetry generators from $H_{n} \sim H_{-n-2}$ should inherit their structure from the Cartan generators of $S U(n+2)$ of the $c=1$ case - precisely as we have shown it for the $n=1$ case. Unfortunately, for $n \geq 2$ the expressions for the Cartan generators become quite cumbersome and we leave the $n \geq 2$ case for the future work. Nevertheless, it seems reasonable to believe that the $\alpha$ symmetry transformations, derived in this paper, are not at all the end of the story. Given the correspondence between the $H_{1} \sim H_{-3} \alpha$-symmetry generators, and the additional symmetries of $2 T$-physics in the case of a particle, the interesting question is whether the list of new symmetries (and, accordingly, the number of the hidden dimensions) observed by Bars, is complete. Superstring theory seems to predict that this list is incomplete, and new hidden symmetries, as well as new space-time dimensions, are yet to be observed. 


\section{References}

[1] H. Verlinde, Phys.Lett. B192:95(1987)

[2] D. Polyakov, Int. Jour. Mod. Phys A20: 2603-2624 (2005)

[3] I.I. Kogan, D. Polyakov, Int.J.Mod.PhysA18:1827(2003)

[4] D. Polyakov, Phys. Rev. D65: 084041 (2002)

[5] D. Polyakov, Int. J. Mod. Phys A20:4001-4020 (2005)

[6] I. Bars, Phys. Rev. D59:045019(1999)

[7] I. Bars, C. Deliduman, D. Minic, Phys.Rev.D59:125004(1999)

[8] I. Bars, C. Deliduman, D. Minic, Phys.Lett.B457:275-284(1999)

[9] I. R. Klebanov, I. I. Kogan, A. M.Polyakov, Phys. Rev. Lett.71:3243-3246 (1993)

[10] B. Lian, G. Zuckerman, Phys.Lett. B254 (1991) 417

[11] I. Klebanov, A. M. Polyakov, Mod.Phys.Lett.A6:3273-3281

[12] E. Witten, Nucl.Phys.B373:187-213 (1992)

[13] D. Polyakov, hep-th/0602209, to appear in IJMPA 